\title{
Design of Armor for Protection against Blast and Impact
}

\author{
Tanaz Rahimzadeh ${ }^{\mathrm{a}}$, Ellen M. Arruda ${ }^{\mathrm{a}, \mathrm{b}, \mathrm{c}, *}$, M. D. Thouless ${ }^{\mathrm{a}, \mathrm{d}, *}$ \\ ${ }^{a}$ Department of Mechanical Engineering, University of Michigan, Ann Arbor, MI \\ 48109, USA \\ ${ }^{b}$ Department of Biomedical Engineering, University of Michigan, Ann Arbor, MI \\ 48109, USA \\ ${ }^{c}$ Macromolecular Science 8 Engineering Program, University of Michigan, Ann \\ Arbor, MI 48109, USA \\ ${ }^{d}$ Department of Materials Science \& Engineering, University of Michigan, Ann \\ Arbor, MI 48109, USA
}

\begin{abstract}
The features of blast and impact that can damage a delicate target supported by a structure include both the peak pressure and the impulse delivered to the structure. This study examines how layers of elastic and visco-elastic materials may be assembled to mitigate these features. The impedance mismatch between two elastic layers is known to reduce the pressure, but dissipation is required to mitigate the transmitted impulse in light-weight armor. A novel design concept called impact or blast tuning is introduced in which a multi-layered armor is used to tune the stress waves resulting from an impact or blast to specific frequencies that match the damping frequencies of visco-elastic layers. The material and geometrical parameters controlling the viscous dissipation of the energy within the armor are identified for a simplified one-dimensional system, to provide insight into how the optimal design of multi-use armor might be based on this concept.
\end{abstract}

Keywords:

Blast mitigation, Armor, Material design, Energy dissipation, Multilayered structures, Viscoelasticity, Frequency tuning, Impact

\footnotetext{
${ }^{*}$ Corresponding authors at: Department of Mechanical Engineering, University of Michigan, Ann Arbor, MI 48109, USA.

Email addresses: arruda@umich.edu, thouless@umich.edu
} 


\section{Introduction}

Pressure waves propagate through a structure subjected to a blast or impact. The mechanics for the two phenomena are similar, although the time scales associated with a blast are significantly smaller. The resultant damage can be of a structural nature if there is collapse or other loss of structural integrity, or of a functional nature if delicate components within a target fail. Furthermore, there are two distinct attributes of pressure waves impinging upon a target that can cause failure: directly-transmitted stresses, and stresses induced by the response to the impulse delivered by the pressure waves. As will be discussed in this paper, the relative importance of the two attributes depends on the characteristic time scale for the dynamic response of the target, compared to that of the impact or blast. Different strategies are needed to mitigate them, and good armor design requires a match between the properties of the target and the armor, and between these and the nature of the threat.

As detailed in the following paragraphs, there has been much work done on the mechanics of blast and its relationship to the optimization of blast-resistant structures. Less work has been done on tailoring material architectures for armor to be used as external protection of delicate structures, such as the brain in a skull. This provides the focus of the current work, in which the mechanics for the mitigation of blast and impact by armor is developed, and then used as the basis for a proposed design for multi-use armor incorporating visco-elastic materials.

The pressure, $P$, exerted on the surface of a structure subjected to a blast or impact rises to a peak value of $P_{o}$, and then decays over time, $t$, so that

$$
P(t)=P_{o} f\left(t / t_{o}\right),
$$


where $t_{0}$ is a characteristic time for the blast or impact. For a blast, the initial rise is almost instantaneous, and $P_{o}$ and $t_{o}$ depend upon the type and mass of explosive material, the distance from the source of the explosion, and the nature of the fluidstructure interaction $[1,2,3,4,5,6,7]$. For an impact, $P_{o}$ and $t_{o}$ depend on the mass, velocity, and mechanical properties of the structure and impacting projectile. The peak pressure is one of the important characteristics of a blast or impact. The other important characteristic is the impulse exerted on the surface of the structure:

$$
I_{0}=\int_{0}^{\infty} P(t) d t
$$

This impulse is responsible for the transfer of kinetic energy which can cause damage to the structure or to the components within it.

Armor plating provides an example of a structure designed to absorb blast-born energy directly, without structural failure. This form of armor can be visualized as clamped plates that undergo bending and stretching in response to the blast, resulting in plastic deformation that dissipates energy $[1,8,9,10,11,12,13,14]$. Additional contributions to energy dissipation can be provided by adding a polymer such as polyurea [15] to the back surface of the plates $[16,17,18]$. An alternative approach for the design of blast-resistant structures is to incorporate an energy-dissipating core, in the form of foams or trusses, between two face-plates. These sandwich structures dissipate energy when the impulse of the pressure wave transmits momentum to a face-plate, which then deforms the core. The structures can be designed with metallic cores and face-plates $[8,19,20,21,22,23,24,25,26,27,28,29,30]$, composite faceplates and polymeric cores [15, 31, 32, 33, 34, 35, 36], or metal-polymer hybrids [37].

Other approaches to dissipate the energy from a blast include the use of air blad- 
ders and fluid-filled chambers [38, 39, 40, 41, 42, 43, 44], granular systems [45, 46, 47], and filled foams [48]. These approaches rely on variations in the mass and stiffness of the components to disperse and attenuate the blast. An active, rather than a passive, approach to blast mitigation has recently been proposed by Wadley et al. [49], using a pre-compressed crushable foam that is relaxed just prior to the arrival of the blast-borne impulse, creating momentum opposing that acquired from the blast.

Studies on the interactions between blast, armor, and targets have been limited to the specific analysis of a combat helmet $[50,51,52,53,54]$, among which a numerical study [50] has shown how the replacement of foam by polyurea as a suspension pad may reduce the peak compressive stress in the brain and its velocity. Beyond this type of empirical study, there has been little focus in the literature on understanding the features of a blast that sensitive targets need to be protected against, and how armor might be optimized to achieve this. This provides the focus for the present paper. The original motivation was provided by design against brain injuries, but it is recognized that sensitive targets requiring protection against blast and impact can be generalized to any dynamical systems, such as those containing MEMS devices [55].

In this paper, we first present a brief discussion on what features of a blast or impact need to be mitigated by armor designed to protect delicate targets, and what approaches can be adopted to do so. We then suggest a novel approach to do this, which involves using a multi-layer design to tune the pressure waves induced by a blast or impulse to the critical damping frequency of a visco-elastic material. By means of this tuning we promote an efficient dissipation of energy. We illustrate this concept of what we call impact or blast tuning by means of a simplified finite-element analysis, and discuss its advantages over other approaches. 


\section{Mechanics of damage from impact and blast}

Recently, there has been much publicity about the damage that can be caused to brain tissue by blast, and by impacts to the head in sports or vehicle crashes. The physiological origin of the damage is not well understood, although it is generally agreed to be the consequence of excessive axonal deformation arising from local deviatoric stresses $[56,57,58]$. Local stresses may also be responsible for the failure of delicate mechanical objects. Therefore, for the purposes of evaluating possible mitigation strategies, it is necessary to identify the important features of any pressure waves induced by blast or impact that may travel through armor to damage a target.

A simplified, but typical, system that might need to be protected from blast and impact can be modelled as a two-component dynamical system consisting of the target and supporting structure. The supporting structure and target are masses coupled by a spring, and the structure is attached to a rigid foundation by a second spring. The motivation for this assumption is a simple model of a head that can be envisaged as a brain coupled to a skull through cerebrospinal fluid, with the motion of the skull being resisted by its attachment to the rest of the body by the neck.

As discussed in the Appendix, the magnitude of the stresses acting on the target in such a system may be controlled either by the magnitude of the pressure wave induced by blast or impact, $P_{o}$, or by the magnitude of the impulse delivered by the pressure wave, $I_{o}$. The relative importance between the two parameters depends on the magnitude of $t_{o}$, the length of the interaction between the pressure pulse and the supporting structure (skull), compared to the characteristic time, $\tau_{\text {target }}$, for the 
dynamic response of the supporting structure and target. If $t_{o}$ is significantly greater than $\tau_{\text {target }}, P_{o}$ is important. Conversely, if $t_{o}$, is significantly less than $\tau_{\text {target }}, I_{o}$ is important.

This important appreciation that either the pressure or the impulse can cause damage was recognized as long ago as 1943 [59], and is reflected in empirical headinjury criteria $[60,61,62]$. However, it is often only the peak acceleration of the supporting structure (skull) that is measured in instrumented studies of impact [63, $64,65,66,67,68]$, assuming that it is only this that acts as a proxy for the damaging stresses. In this work, we will focus on how both the pressure and the impulse transmitted to a structure can be mitigated by armor.

\section{Blast mitigation approaches}

The role of well-designed armor is to ensure that the magnitude of the pressure wave and its impulse, and the length of the interaction time between the pressure wave and the structure, are controlled to keep any resultant stresses acting on the target below a critical threshold. This may be done by adjusting any, or all, of the three parameters, depending on the nature of the threat and the properties of the target. In this section, we will summarize the general strategies that can be used in armor design. It is important to appreciate that, in this paper, we ignore the role that the layer of armor facing the threat may have on determining the pressure waves that travel through it. This will be addressed elsewhere for impact studies.

Impedance-mismatch can be used to mitigate the peak pressure transmitted through armor. If an incident compressive stress wave of amplitude $\sigma_{i}$ traveling in an elastic 
material $A$ passes through an interface with another elastic material $B$ that is normal to the direction of propagation, the amplitude of the transmitted compressive stress wave, $\sigma_{t}$ in $B$ is given by [69]

$$
\sigma_{t}=\frac{2 \sqrt{E_{B} \rho_{B}}}{\sqrt{E_{A} \rho_{A}}+\sqrt{E_{B} \rho_{B}}} \sigma_{i},
$$

where $E$ is the modulus, $\rho$ is the density, and the subscripts $A$ and $B$ indicate the two materials. As indicated by this equation, the magnitude of a transmitted stress wave is reduced by making the acoustic impedance, defined as $\sqrt{E \rho}$, of the second material much smaller than that of the first material.

The transmitted impulse is affected by energy dissipation within the armor, and by the relative masses of the armor and protected system. If there is no energy dissipation, a simple, rigid-body-dynamics analysis indicates that the ratio between the impulse transmitted to the structure, $I_{t}$, and the original impulse imparted to the armor, $I_{o}$, is $[70]$

$$
\frac{I_{t}}{I_{o}}=\frac{2\left(m_{s} / m_{a}\right)}{\left(m_{s} / m_{a}\right)+1},
$$

where $m_{a}$ is the mass of the armor, and $m_{s}$ is the mass of the structure and target. Therefore, one approach for mitigating impulse is to use massive armor $\left(m_{a}>>m_{s}\right)$.

An approach that relies on heavy armor conflicts with a common design criterion of minimizing mass. An alternative approach is to reduce the transmitted impulse by dissipating energy within the armor. Rigid-body dynamics shows that there is a limit to this approach, in that the minimum ratio for $I_{t} / I_{o}$, corresponding to a perfectly-inelastic collision, is given by [70]

$$
\frac{I_{t}}{I_{o}}=\frac{\left(m_{s} / m_{a}\right)}{\left(m_{s} / m_{a}\right)+1} .
$$


If this reduction in impulse is not enough to protect the target, an alternative design approach is needed: either more massive armor, or an increase in the interaction time $\left(t_{o}\right)$ to move the design space to one in which it is the pressure, not the impulse, that needs to be mitigated (see Appendix).

Energy can be dissipated in armor using mechanisms such as plasticity and viscoelasticity, as well as friction and delamination. Plastic deformation and delamination are irreversible, and are limited to a single use. For example, once an element of material has plastically deformed, it is unable to dissipate energy from subsequent stress waves of the same magnitude. Visco-elastic deformation can dissipate energy over multiple cycles. However, the efficiency with which energy is dissipated within visco-elastic materials depends on the amplitudes and frequencies of any stress waves traveling through them. In general, the stresses induced by a blast or impact exhibit a broad range of frequency components, only a few of which will be associated with the optimal damping of a particular visco-elastic material. Therefore, the introduction of a layer of visco-elastic material into armor, without thought about its characteristic frequencies, may not be useful. This consideration led us to a novel concept we call impact or blast tuning, in which a multi-layer construction is used to tune the stresses induced by impacts and blasts to frequencies that can be matched to the optimal energy-dissipating frequencies of a visco-elastic layer.

An additional consideration raised by Eqns. 4 and 5 is that there is a limit to the possible reduction in transmitted impulse, which depends on the mass of the armor. However, as mentioned above, if the time scale of a pressure pulse is increased it may be possible to move the design space out of the regime in which damage is correlated to impulse. This may be achieved as a direct consequence of the conservation 
of momentum if the average pressure of a pulse is lowered. It may also be achieved in visco-elastic materials as a consequence of dispersion associated with frequencydependent elastic properties. While there are effects of dispersion in the approach we propose, it is important to appreciate that our approach relies primarily on dissipation.

\section{The concept of tuning}

In this section, the principles of linear visco-elasticity are used to introduce the concept of tuning. We assume that a visco-elastic material can be represented as a standard-linear solid (SLS), consisting of a linear-elastic spring in parallel with a Maxwell element (a linear-elastic spring and a linear-viscous damper connected in series). The parameters describing the constitutive properties are the unrelaxed modulus, $E_{u}$, the relaxed modulus, $E_{r}$, and the relaxation time, $\tau$. (In this paper, the use of the word "modulus" for a polymer always implies the "storage modulus" or the "real part" of the complex modulus.) If such a material is subjected to an oscillating stress at an angular frequency $\omega$, the ratio of the loss modulus to the storage modulus is given by $[71]$

$$
\tan \delta=\frac{\left(E_{u}-E_{r}\right) \tau \omega}{\tau^{2} \omega^{2} E_{u}+E_{r}}
$$

The energy dissipated in the material scales with $\tan \delta$. The maximum value of this quantity is given by

$$
(\tan \delta)_{\max }=\frac{E_{u}-E_{r}}{2 \sqrt{E_{r} E_{u}}}
$$

at a critical frequency of $[71]$

$$
\omega_{\text {crit }}=\frac{\sqrt{E_{r} / E_{u}}}{\tau}=2 \pi f_{\text {crit }} .
$$


Hence, it can be seen that an optimally dissipative material is one in which the frequency of the stress wave matches the critical frequency, and the difference in the unrelaxed and relaxed moduli is as large as possible.

The problem with using visco-elastic materials to dissipate the energy of a blast (or impulsive impact) is that the impulse is usually delivered to the armor in the form of a single pulse, which induces stress waves that are represented by a wide range of frequencies in the Fourier domain. Both of these considerations, the single pulse and the wide range of characteristic frequencies, might appear to make viscoelastic materials unattractive for energy dissipation in this application. However, as explained below, it is possible to use a multi-layered structure to tune the stresses to characteristic frequencies that can then be dissipated by an appropriate choice of visco-elastic materials.

To achieve the tuning required to realize optimal damping, two layers of materials need to be placed between the threat and the visco-elastic layer. The first layer, closest to the threat, needs to be stiff and dense compared to the second layer, so as to ensure a large mismatch in acoustic impedance between the two layers. The internal reflections within the first layer will tune the stresses to a characteristic frequency of

$$
f_{A}=\frac{\sqrt{E_{1} / \rho_{1}}}{2 L_{1}} ;
$$

where $E_{1}, \rho_{1}$, and $L_{1}$ are the modulus (assumed to be isotropic here), density, and thickness of the first layer. The pressure waves at this frequency that subsequently enter the visco-elastic layer will be efficiently dissipated if $f_{A}$ matches $f_{\text {crit }}$ (Eqn. 8). 
The energy dissipated in a visco-elastic material increases with the number of loading and unloading cycles, and with their amplitude. This adds some additional considerations to the design of multi-layered armor. First, the impedance mismatch between the first and second layers must be just large enough to provide good tuning without excessively reducing the amplitude of the stresses that eventually get transmitted into the energy-absorbing layer. For a similar reason, to ensure a reasonable amplitude of stress waves for dissipation, the impedance of the third layer must be relatively high compared to the impedance of the second layer. Furthermore, the need to have many stress cycles within the visco-elastic layer suggests that the tuned frequency should be as high as possible, consistent with finding a suitable corresponding visco-elastic material. The higher the frequency, the less material is needed to dissipate the energy (for a given wave speed). The potential materials challenge from a development perspective is that the frequencies associated with this tuning will be in a range for which the properties of polymers have only received limited study $[72,73,74,75]$.

It was noted from the results of the finite-element analyses described in the next section that, in addition to $f_{A}$, two other, significantly lower, characteristic frequencies, $f_{B}$ and $f_{C}$, enter the visco-elastic layer in a tuned system. These other two frequencies have periods corresponding to the times for the tuned wave to traverse the third and second layers, respectively. Spectral analyses of the waves in the first element of the visco-elastic material indicated that, for a typical well-designed system, about $45 \%$ of the impulse was carried by $f_{A}$, about $40 \%$ was carried by $f_{B}$, and about $15 \%$ was carried by $f_{C}$ and its harmonics.

In the present implementation, we have analyzed a system with only one critical 
frequency, so only the high-frequency, $f_{A}$, component is dissipated. In principle, one might consider implementing a design with additional visco-elastic materials, or with a material having multiple relaxation times, to mitigate the other frequencies. However, it should be noted that, even if the lower frequencies are not dissipated, they serve a useful purpose by transmitting some of the impulse through the armor at a lower velocity. As discussed in Section 2, one strategy for blast and impact mitigation is to increase the transmission time so that the peak pressure (which can then be controlled by impedance mismatch), rather than impulse, dominates the response of the target.

\section{Finite-element analysis of blast mitigation}

In this paper, we used a commercial finite-element code, ABAQUS Explicit [76], to analyze blast mitigation. In these analyses, the internal interactions within the protected structure, between the supporting structure and the delicate target, were not addressed. It was assumed that the characteristics of the pressure pulse that the structure can support without damage to the target are known from a separate analysis of the protected structure. We, therefore, compared the maximum amplitude of the stress wave, $P_{t}$, and impulse, $I_{t}$, transmitted through the armor to an elastic structure behind it, to the corresponding values of $P_{o}$ and $I_{o}$ of the original pressure pulse applied to the surface of the armor. The relative ratios of $P_{t} / P_{o}$ and $I_{t} / I_{o}$ were taken as the two primary measures of performance of the armor, and used for comparisons between different designs.

The armor and the protected structure were modeled using three-dimensional, eight-node brick elements with reduced integration. The geometry is shown in Fig. 1. 


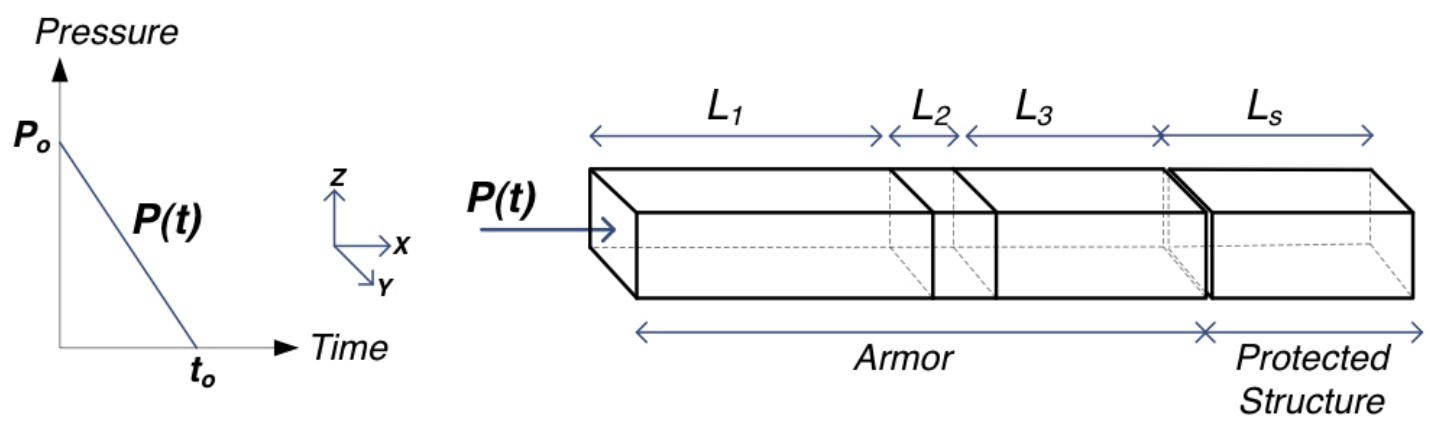

Figure 1: Geometry of the armor and protected structure exposed to a time-varying normal load, $P(t)$, analyzed in the finite-element model.

The displacements along one set of $x z$ - and $x y$-faces were constrained in the $y$ - and $z$ directions, respectively. The other $x z$ - and $x y$-faces were traction-free. The interfaces between the internal layers of the armor were bonded. The protected structure was a solid, linear-elastic block. The interface between the structure and the armor was frictionless. A pressure $P(t)$ was applied to the external surface of the armor, along the $x$-direction (Fig. 1). This pressure decayed linearly to zero from an initial value of $P_{o}$ during a time $t_{o}$. It was verified that the major conclusions of the study were not sensitive to this particular choice of $P(t)$.

The peak pressure transmitted to the protected structure, $P_{t}$, was taken to be the maximum value of the longitudinal stress at the internal surface of the armor, as calculated from the finite-element calculations. Determining the transmitted impulse was more complicated. As will be discussed in more detail later, two distinct types of behavior were observed. In one type of behavior, momentum was transferred to the structure/target system in a single broad pulse. In the other type of behavior, momentum was transferred over a large number of broad pulses separated by significant periods of time. Owing to the time scales involved, only the impulse delivered in the first broad pulse was considered. Therefore, rather than comparing $I_{o}$ to the total 
transmitted impulse, we compared it to the effective transmitted impulse $I_{\text {eff }}$, determined by integrating the longitudinal stress at the internal surface of the armor over the first broad pulse delivered to the structure. The calculations for the transmitted impulse were verified by comparing it to the momentum of the protected structure.

Since a commercial code was used, considerable care was taken to reduce the effects of any spurious numerical artifacts to below an acceptable level of numerical uncertainty that we indicate on our plots. For example, the default bulk-viscosity parameter was set to zero, and double-precision was used for the solutions. Additional concerns were that the effects of the two non-dimensional groups involving the timestep and mesh size should be no larger than the uncertainties introduced by any of the other unspecified non-dimensional groups (see Section 6). We did this by ensuring an adequate number of time steps and nodes for the highest frequency and shortest wavelength of interest. To satisfy the first requirement, the highest frequency seen in any of the three layers and its associated period were identified, and the time increment was set to be no more than $20 \%$ of this period. To satisfy the second requirement, the shortest wavelength in a simulation was identified, and the largest element size was set to be no more than $20 \%$ of that wavelength. We also ensured that the CourantFriedriches-Lewy condition was met by setting the Courant number to 0.2. Finally, we verified that any changes introduced in the solutions by further spacial or temporal refinement were insignificant within the limits of the quoted error bars. Examples of the form of the stress waves at different locations within the armor are given in Fig. 2. 


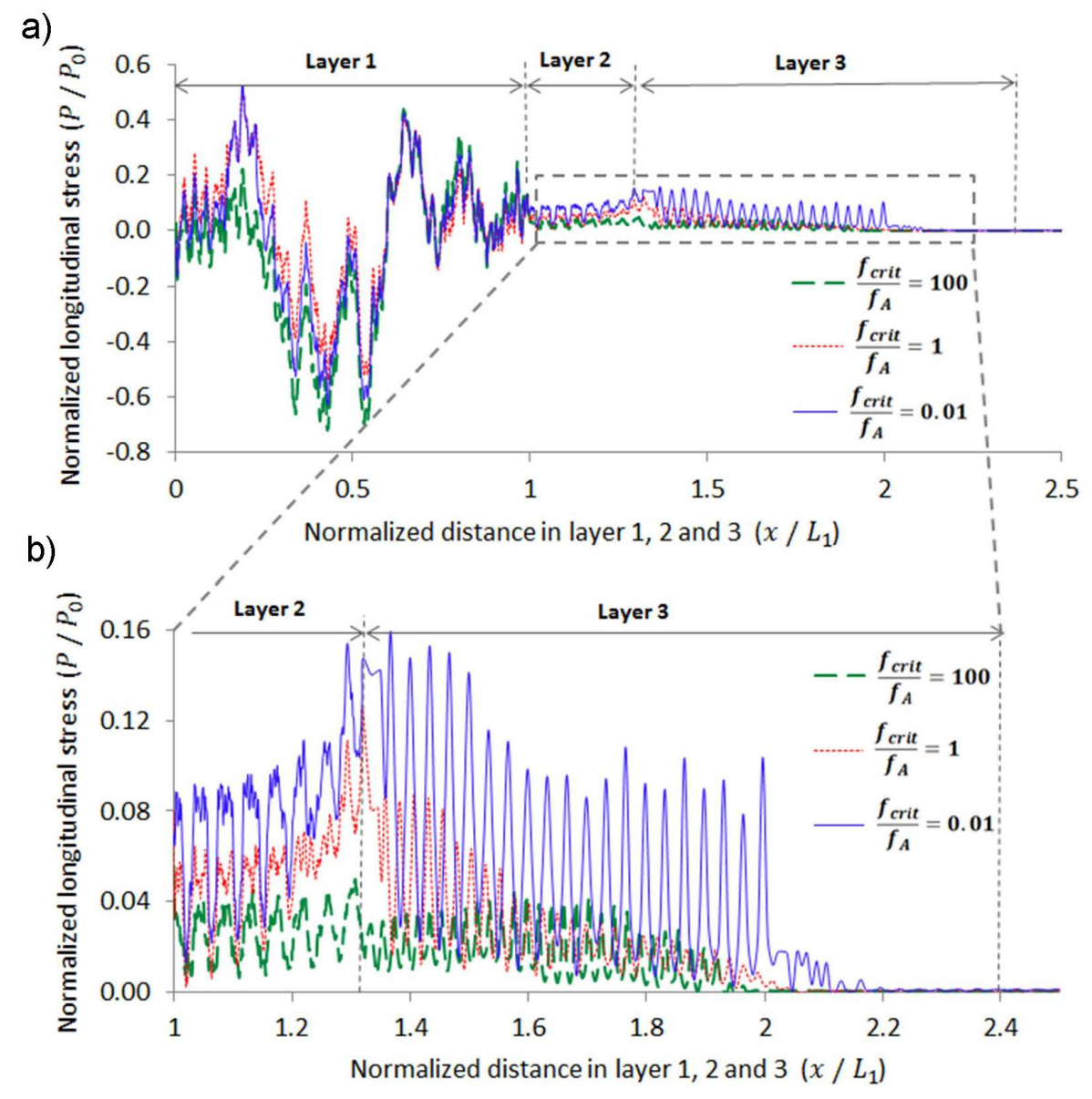

Figure 2: An example of a spatial plot showing how the magnitudes of the stress waves vary as a function of distance through the armor for a well-tuned system $\left(f_{\text {crit }} / f_{A}=1\right)$, and two poorly-tuned systems $\left(f_{\text {crit }} / f_{A}=0.01\right.$ and 100). (b) shows a detail of (a). These plots were taken at a normalized time of $f_{A} t=46$. The values of the other groups are given by $E_{\text {crit }} \rho_{3} / E_{2} \rho_{2}=1, E_{r} / E_{u}=0.001$ and $t_{3} / t_{1}=100$. The non-dimensional groups are explained in Section 6 . 


\section{Analysis of the three-layered armor}

In the analyses of the armor, it was assumed that each layer was isotropic. Layer 1 had a Young's modulus of $E_{1}$, a density of $\rho_{1}$, and a length of $L_{1}$. Layer 2 had a Young's modulus, of $E_{2}$, a density of $\rho_{2}$, and a length of $L_{2}$. The visco-elastic layer was modeled as a standard-linear solid with an unrelaxed modulus of $E_{u}$, a relaxed modulus of $E_{r}$, a characteristic relaxation time of $\tau$, a density of $\rho_{3}$, and a length of $L_{3}$. The plate representing the supporting structure had a Young's modulus of $E_{s}$, a density of $\rho_{s}$, and a mass of $m_{s}$. These dimensions and properties, plus the two characteristics of the blast, $P_{o}$ and $t_{o}$, make a total of sixteen variables and three different units to describe the propagation of the stress wave through the armor. Therefore, according to the Buckingham- $\Pi$ theory $[77,78,79]$, the resultant impulse and pressure must be a function of thirteen dimensionless groups.

A series of finite-element calculations indicated that seven groups could be reasonably neglected for the calculations that are presented, leaving $I_{\text {eff }}$ and $P_{t}$ as functions of six dimensionless groups:

$$
\begin{aligned}
& \frac{I_{\text {eff }}}{I_{o}}=f\left(\frac{E_{1} \rho_{1}}{E_{2} \rho_{2}}, \frac{m_{s}}{\rho_{1} L_{1}+\rho_{2} L_{2}+\rho_{3} L_{3}}, \frac{f_{\text {crit }}}{f_{A}}, \frac{t_{3}}{t_{1}}, \frac{E_{\text {crit }} \rho_{3}}{E_{2} \rho_{2}}, \frac{E_{r}}{E_{u}}\right) \\
& \frac{P_{t}}{P_{o}}=f\left(\frac{E_{1} \rho_{1}}{E_{2} \rho_{2}}, \frac{m_{s}}{\rho_{1} L_{1}+\rho_{2} L_{2}+\rho_{3} L_{3}}, \frac{f_{\text {crit }}}{f_{A}}, \frac{t_{3}}{t_{1}}, \frac{E_{\text {crit }} \rho_{3}}{E_{2} \rho_{2}}, \frac{E_{r}}{E_{u}}\right) .
\end{aligned}
$$

In these expressions, the critical frequency, $f_{c r i t}$, and the tuned frequency, $f_{A}$, have been defined in Eqns. 8 and 9. $E_{\text {crit }}$ is the storage modulus of the third layer at the

critical frequency [71], $t_{1}$ is the time for a stress wave to travel through layer 1 , and $t_{3}$ is the time for a stress wave at the critical frequency to travel through layer 3 :

$$
\begin{aligned}
E_{c r i t} & =\left(2 E_{u} E_{r}\right) /\left(E_{u}+E_{r}\right) \\
t_{1} & =L_{1} \sqrt{\rho_{1} / E_{1}}=1 / 2 f_{A}
\end{aligned}
$$




$$
t_{3}=L_{3} \sqrt{\rho_{3} / E_{\text {crit }}}
$$

The first non-dimensional group, $E_{1} \rho_{1} / E_{2} \rho_{2}$, is the square of the impedance mismatch between the first two layers. As discussed earlier, this needs to be high enough to ensure tuning, but low enough to make sure that the stress waves pass through to the visco-elastic layer where they can be damped. A series of finite-element calculations indicated that the optimal level for the impedance mismatch is about 70 . So, in all the calculations that follow, $E_{1} \rho_{1} / E_{2} \rho_{2}$ was set to a fixed value of 5,000 . The second non-dimensional group is the relative mass of the protected structure to the mass of the armor. As indicated in Section 3, the transmitted impulse decreases with the relative mass of the armor. Therefore, to provide a point of comparison between the different calculations, $m_{s} /\left(\rho_{1} L_{1}+\rho_{2} L_{2}+\rho_{3} L_{3}\right)=1$ for all calculations. The effects of the other four important non-dimensional groups will be seen in the results that follow. During the calculations, the remaining seven, non-critical, groups were allowed to vary within fairly broad ranges ${ }^{1}$. The error bars shown on the figures are the result of both numerical uncertainties and the variations of these non-critical groups.

In the results that follow, it will be observed that there are regimes in which the transmitted impulse appears to be less than $0.5 I_{o}$ : less than that expected to be transmitted by perfectly-plastic armor. This can be explained by reference to Fig. 3, which shows how the pressure can be transmitted to the protected structure through widely spaced pulses. Poorly-tuned armor transmits the impulse over a single broad

\footnotetext{
${ }^{1} 0.1<t_{o} / t_{1}<10,0.08<L_{1} / L_{2}<10,5<E_{1} / E_{2}<25,000,7 \times 10^{-5}<E_{\text {crit }} / E_{2}<60$, $E_{s} \rho_{s} / E_{\text {crit }} \rho_{3}>8,000,0.8<\rho_{s} / \rho_{3}<2$, and $10^{-5}<P_{o} / E_{1}<0.4$.
} 
pulse. Good tuning and dissipation results in only some of the impulse being transmitted in the first pulse before contact is lost with the protected structure; the rest of the impulse is then transmitted when there is subsequent contact, sometimes after multiple internal reflections within the armor. The impulse transmitted to the armor in the initial pulse is defined as the "effective" impulse, $I_{e f f}$, since the subsequent pulses occur so much later that they will probably have no significant effect on the target (see Appendix).

A well-tuned wave is expected to be optimally damped at $f_{\text {crit }} / f_{A}=1$, and the effectiveness of the damping is expected to increase with the number of cycles that the pressure wave experiences in the visco-elastic layer. The first concept is illustrated by the plots in Figs. 4 and 5 that show minima in the effective impulse and transmitted pressure at $f_{\text {crit }} / f_{A}=1$. The second concept is illustrated by the plots in Figs. 6 and 7 that show how the effective impulse and transmitted pressure decrease as the time for the stress wave to traverse the visco-elastic layer is increased. This travel time can be increased either by increasing the thickness of the visco-elastic layer, or by decreasing the wave speed.

Figures 4 and 6 show that there is no significant reduction in the effective impulse if $E_{r} / E_{u} \geq 0.1$, because there is no energy dissipation, even for well-tuned armor. Correspondingly, the drop in the amplitude of the transmitted pressure for $E_{r} / E_{u} \geq 0.1$ shown in Figs. 5 and 7 is determined only by impedance mismatch. Finally, it will be noted from the plots in Figs. 4 and 5 that, when $E_{r} / E_{u}$ is small, the minima are fairly broad for values of $f_{c r i t} / f_{A}>1$. This can be explained by reference to Eqns. 6 and 8; as $E_{r} / E_{u}$ becomes smaller, $\tan \delta$ exhibits a broader peak 

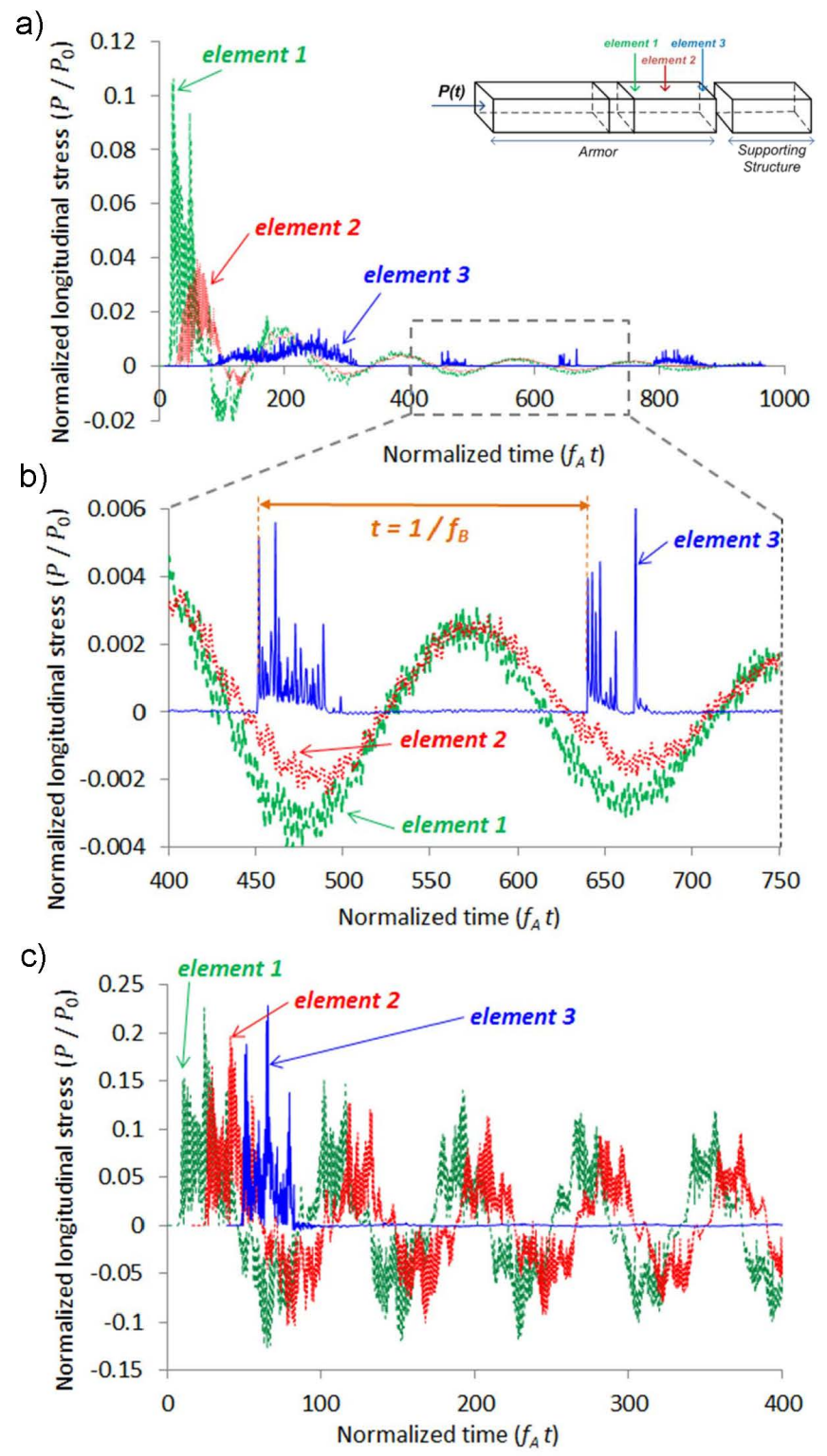

Figure 3: Pressure waves at the beginning, middle, and end of the visco-elastic layer. These are shown in (a) and (b) for a well-tuned system with $f_{A} / f_{\text {crit }}=1$, and in (c) for a poorly-tuned system with $f_{A} / f_{\text {crit }}=0.01$. The values of the other significant groups are given by $E_{\text {crit }} \rho_{3} / E_{2} \rho_{2}=1$, $E_{r} / E_{u}=0.001$ and $t_{3} / t_{1}=100$. A detail of the stress waves for (a) is shown in (b). These figures show how, in a well-tuned system, the latter parts of the impulse can be transmitted to the protected structure in pulses separated by time intervals that are increments of $1 / f_{B}$. While in a poorly-tuned system, the impulse gets transmitted to the protected structure over a single broad pulse. 


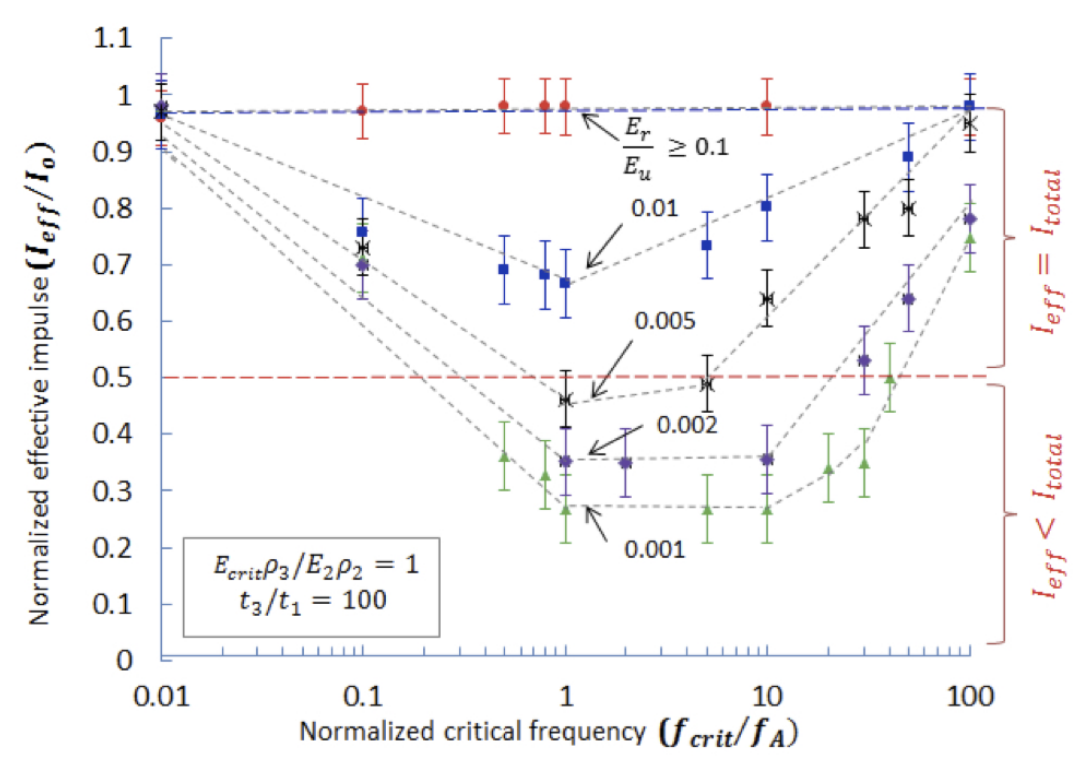

Figure 4: The effective impulse is minimized after passage through a three-layer visco-elastic armor when $f_{\text {crit }} / f_{A}=1$. The transmitted impulse occurs in a single pulse for $E_{r} / E_{u}>0.005$. For lower values of $E_{r} / E_{u}$, the impulse is transmitted in a series of pulses, so $I_{\text {eff }}<I_{o} / 2$. When $E_{r} / E_{u}>0.1$, there is negligible dissipation in the armor, and there is no reduction in the transmitted impulse.

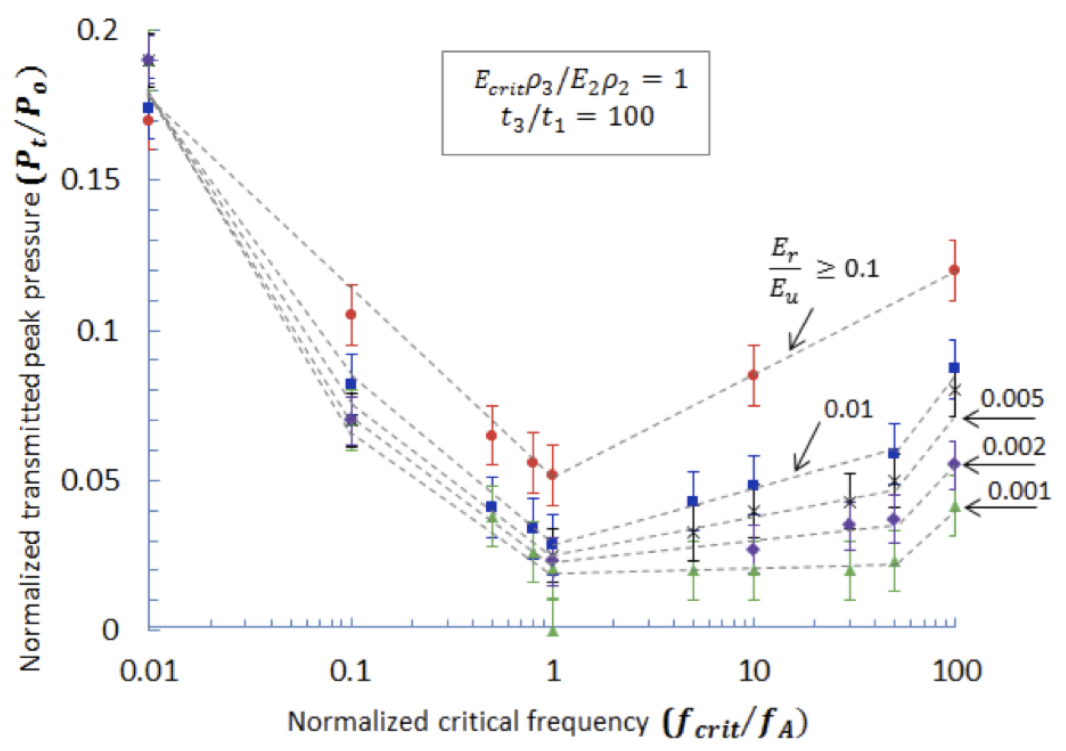

Figure 5: A significant reduction in the transmitted peak pressure can be achieved with a well-tuned, three-layer visco-elastic armor. The reduction in this peak pressure is increased as the ratio $E_{r} / E_{u}$ is reduced. When $E_{r} / E_{u}>0.1$, there is negligible dissipation in the armor, and any reduction in the pressure pulse occurs only because of impedance mismatch. 


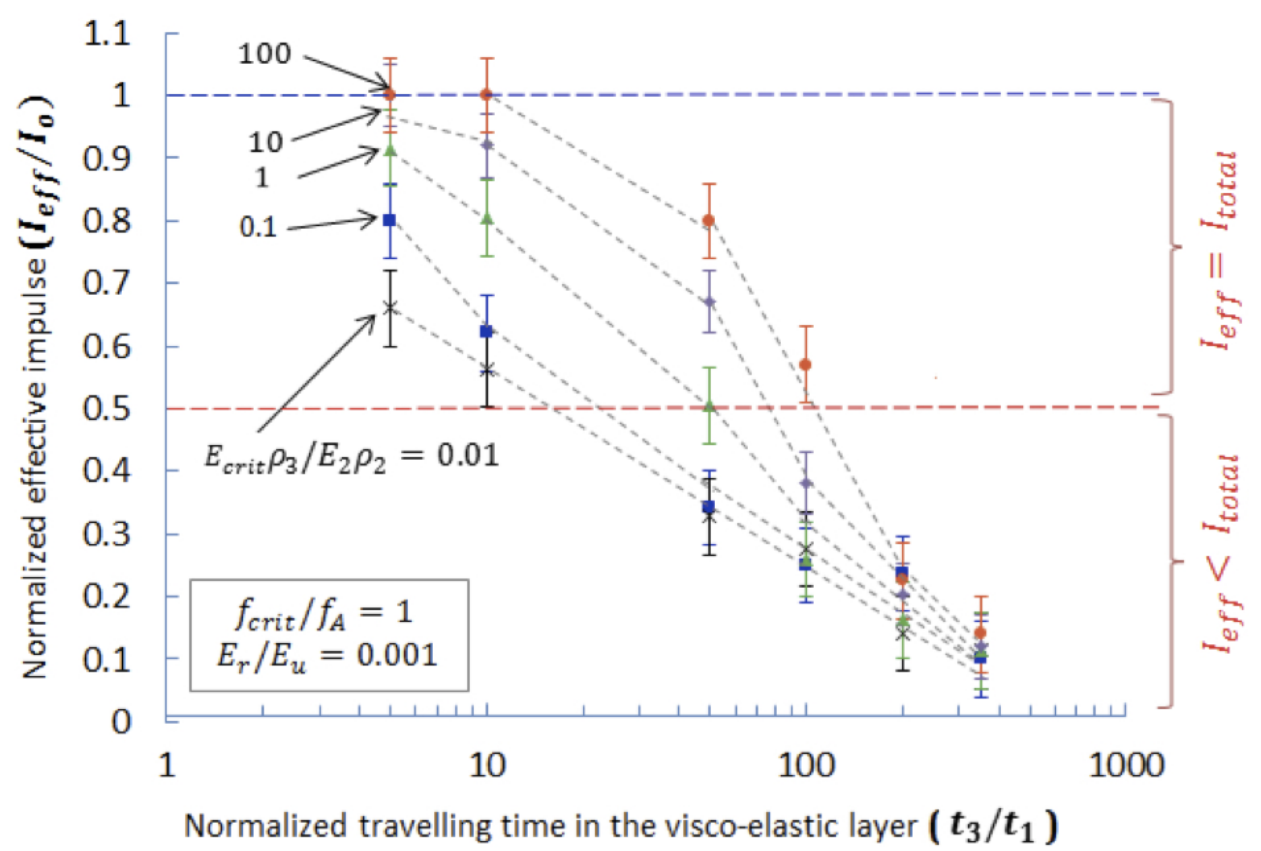

Figure 6: For a well-tuned three-layer armor, the effective impulse drops with the time taken for the stress wave to traverse the visco-elastic layer.

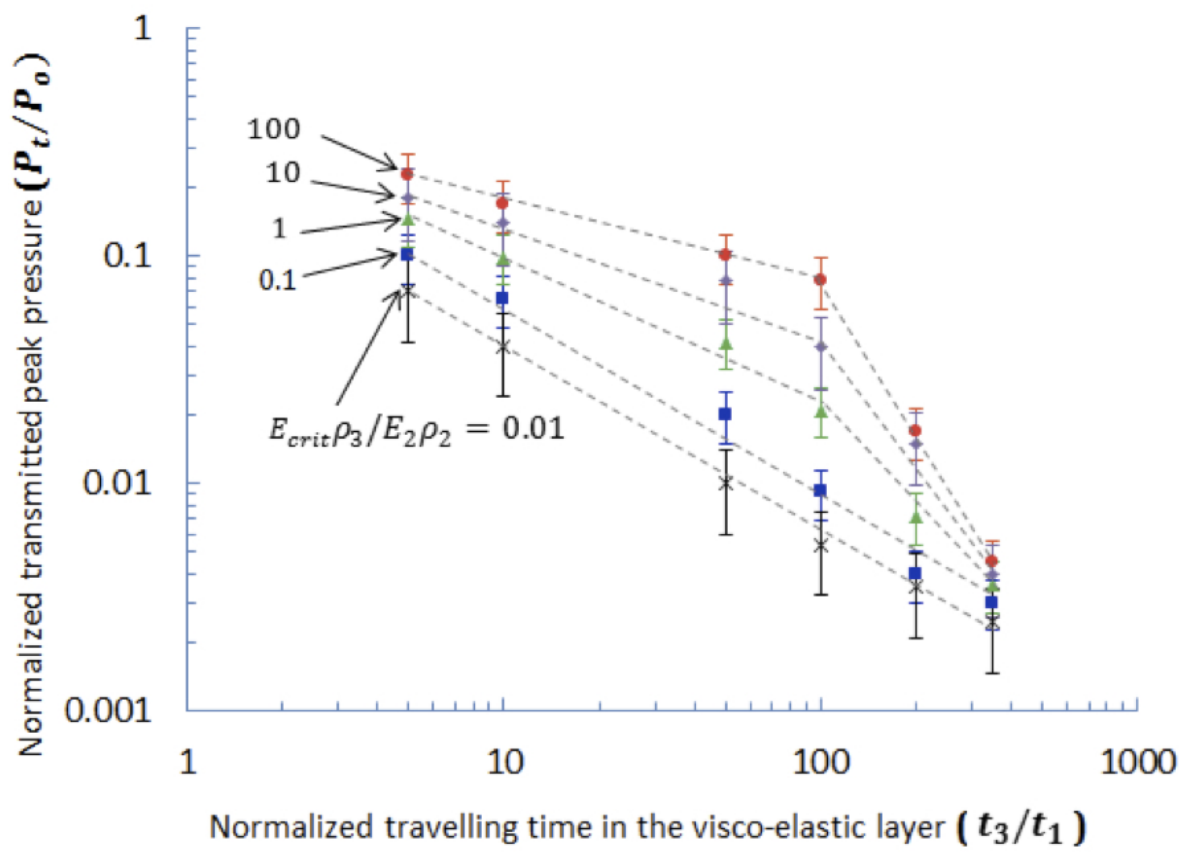

Figure 7: For a well-tuned three-layer armor, the maximum amplitude of the transmitted pressure drops with the time taken for the stress wave to traverse the visco-elastic layer, since this allows an increase in energy dissipation. 
skewed to $f_{\text {crit }} / f_{A}>1$. As will be discussed later, this may have significant practical importance from a design perspective in reducing the sensitivity of the performance of the armor to variations in operating conditions.

\section{Comparison with elastic and plastic designs}

Armor consisting of only elastic layers will transmit an impulse equal to the initial impulse, if it has the same mass as the supporting structure. The transmitted pressure in such a system can be controlled using impedance mismatch between the two layers. Furthermore, a second layer with a low impedance could increase the time-scale for the transmission of the impulse sufficiently to make control of the pressure amplitudes a more important design consideration than control of impulse. For cheap, low-performance armor and helmets, this approach certainly provides a possible design strategy. Whether it is suitable or not, would depend on the application.

Armor containing a plastic layer to dissipate energy would result in the impulse always being transmitted over a single broad pulse. If the armor and supporting structure have equal mass, the magnitude of the transmitted impulse would be in the range $I_{o}$ to $0.5 I_{o}$, depending on how efficiently the armor dissipates energy. Furthermore, although energy can be as dissipated with plasticity as efficiently as with visco-elasticity, the use of plastic armor is limited to a single incident.

\section{Conclusions}

The stress induced within a target that is part of a dynamical system, such as the brain within a skull, is determined either by the directly transmitted pressure, 
or by the transmitted impulse. The relative importance of the two depends on the duration of the pressure wave impinging upon the protected structure, compared to the characteristic time of the dynamic response of the system. The design of any armor used as protection from blast in military applications, or by impact in sporting or industrial applications, needs to consider both of these time scales. Armor can mitigate both the pressure and the impulse. It can also change the time scale over which a pressure wave is transmitted, so as to move the design away from one in which impulse needs to be mitigated, to one in which the pressure needs to be limited. Impedance mismatch can control the transmitted pressure. Energy dissipation mechanisms can mitigate the transmitted impulse. Dispersion (and impedance mismatch) can increase the interaction time between the supported structure and the threat, so as to change the damage regime from one controlled by impulse to one controlled by pressure.

In this paper we have proposed that visco-elastic polymers can be used for protection over multiple events. However, for this concept to be realized, it is necessary for the stress waves traveling through the armor to do so at frequencies corresponding to appropriate dissipative molecular transitions in the polymers. Typically, the energy of the stress waves induced by blast or impact is broadly distributed over multiple frequencies. Therefore, this energy must be tuned to a narrow spectrum before it can be optimally dissipated by the polymer. Here, it is proposed to do this through a multi-layer design in which the outer layers tune the stress waves to match the critical damping frequency of the inner visco-elastic layer. As a high-frequency stress wave travels through this visco-elastic layer, it undergoes multiple loading-unloading cycles which can result in significant energy dissipation over a short duration. 
A finite-element analysis of this concept has illustrated several important constraints on the design. The outer layer needs to have a high acoustic impedance compared to its neighbor, so that the wave can be tuned by multiple reflections at the interface between the two layers. Although, for reasons discussed in Section 6, the impedance mismatch must not be so high that the stress waves is transmitted inefficiently through successive layers. The numerical simulations suggest that an impedance mismatch of about 70, is optimal. Typically, one would expect to use an outer layer with a relatively high modulus; but it is recognized that the outer layer may also have to serve other functional purposes, such as resistance to ballistic penetration. It is also recognized that in sports applications involving impact, more compliant helmets may increase the characteristic time for transmission of an impulse, and move the design space to a regime where dissipation of energy is less critical.

There are two significant constraints on the material properties of the visco-elastic layer used for energy dissipation. First, it needs to have a very low ratio of the relaxed modulus to the unrelaxed modulus. This will result in a high value of $\tan \delta$ at a critical frequency that matches the tuned frequency from the first layer. Second, for realistic thicknesses of the outer layer, the critical frequency of the polymer will need to be quite high. For example, if the modulus of the outer layer is of the order of 1-10 GPa, its density is $1000 \mathrm{~kg} \cdot \mathrm{m}^{-3}$, and its length is $5 \mathrm{~mm}$, the critical frequency that needs to be damped will be about 100-300 kHz. This leads to some experimental challenges in identifying and designing suitable polymers, for it is far above the range of frequencies at which polymers are typically investigated. However, ultrasonic methods [72] and dielectric analyses [75] have been used to measure tan $\delta$ and the storage and loss moduli in the $\mathrm{MHz}$ range. 
The glass transition is a possible energy-loss peak to explore for these purposes [73, 74], and it is instructive to consider what value of glass-transition temperature, $T_{g}$, measured in the $1 \mathrm{~Hz}$ range, might correspond to a glass transition in the $200 \mathrm{kHz}$ range at an operating temperature of $T_{o}$. This can be estimated from the WLF equation [80], for a shift factor of $2 \times 10^{5}$ :

$$
\log \left(2 \times 10^{5}\right)=\frac{17.5\left(T_{o}-T_{g}\right)}{52+T_{o}-T_{g}}
$$

This expression results in a glass transition temperature measured at $1 \mathrm{~Hz}$ which is about $23{ }^{\circ} \mathrm{C}$ below the operating temperature. For example, if the inside of a helmet is maintained at body temperature, the required glass-transition temperature would be about $14^{\circ} \mathrm{C}$. However, there will obviously be a range of operating temperatures, depending on the external environment. Fortunately, the analysis (Figs. 4 and 5) shows that the efficacy of the proposed design is relatively insensitive to $f_{\text {crit }}$, provided that $f_{\text {crit }} / f_{A}>1$ and $E_{r} / E_{u}$ is very small. In practice, this puts an upper bound on $T_{g}$ of about $20-30{ }^{\circ} \mathrm{C}$ below the lowest temperature that the armor will ex-

perience in service. Higher temperatures, within a reasonable range, will result in the system being within the plateau where the response is not very sensitive to $f_{\text {crit }} / f_{A}$. In conclusion, this result would seem to indicate that there is plenty of flexibility to use even higher tuning frequencies than $200 \mathrm{kHz}$, since the required value of $T_{g}$ is not particularly low.

\section{Acknowledgements}

This work has been supported by the ONR under grant ONR N00014-10-1-415. The authors are grateful for many helpful discussions with Prof. A. M. Waas during the course of this project. 


\section{References}

[1] G.I. Taylor. Pressure and impulse of submarine explosion waves on plates. In G.K. Batchelor, editor, The Scientific Papers of Sir Geoffrey Ingram Taylor, Volume III: Aerodynamics and the Mechanics of Projectiles and Explosions, pages 287-303. Cambridge University Press, Cambridge, UK, 1963.

[2] N. Kambouchev, R. Radovitzky, and L. Noels. Fluid-structure interaction effects in the dynamic response of free-standing plates to uniform shock loading. Journal of Applied Mechanics, 74(5):1042-1045, 2007.

[3] N. Kambouchev, L. Noels, and R. Radovitzky. Nonlinear compressibility effects in fluid-structure interaction and their implications on the air-blast loading of structures. Journal of Applied Physics, 100(063519):1-12, 2006.

[4] N. Kambouchev, L. Noels, and R. Radovitzky. Numerical simulation of the fluidstructure interaction between air blast waves and free-standing plates. Computers Es Structures, 85(11-14):923-931, 2007.

[5] P.D. Smith and J.G. Hetherington. Blast and Ballistic Loading of Structures. Butterworth Heinemann, Burlington, MA, USA, 1994.

[6] M.M. Swisdak. Explosion effects and properties: Part II - Explosion effects in water. Technical Report NSWCIWOL TR 76-116, Naval Surface Weapons Center, White Oak, Silver Spring, MD 20910, USA, 1978.

[7] M.F. Ashby, A.G. Evans, N.A. Fleck, L.J. Gibson, J.W. Hutchinson, and H.N.G. Wadley. Metal Foams: A Design Guide. Butterworth Heinemann, Woburn, MA, USA, 2000. 
[8] Z. Xue and J.W. Hutchinson. A comparative study of impulse-resistant metal sandwich plates. International Journal of Impact Engineering, 30(10):1283-1305, 2004.

[9] A.J. Wang and H.G. Hopkins. On the plastic deformation of built-in circular plates under impulsive load. Journal of the Mechanics and Physics of Solids, $8: 22-87,1954$.

[10] A.L. Florence. Clamped circular rigid-plastic plates under central blast loading. International Journal of Solids Structures, 2:319-335, 1966.

[11] P.S. Symonds and T. Wierzbicki. Membrane mode solutions for impulsively loaded circular plates. Journal of Applied Mechanics, 46(1):58-64, 1979.

[12] N. Jones. A theoretical study of the dynamic plastic behaviour of beams and plates with finite-deflections. International Journal of Solids and Structures, $7(8): 1007-1029,1971$.

[13] N. Jones. Structural Impact. Cambridge University Press, Cambridge, UK, 2nd edition, 1989.

[14] Z. Xue and J.W. Hutchinson. Preliminary assessment of sandwich plates subject to blast loads. International Journal of Mechanical Sciences, 45(4):687-705, 2003.

[15] A.V. Amirkhizi, J. Isaacs, J. McGee, and S. Nemat-Nasser. An experimentallybased viscoelastic constitutive model for polyurea, including pressure and temperature effects. Philosophical Magazine, 86(36):5847-5866, 2006.

[16] M.R. Amini, A.V. Amirkhizi, and S. Nemat-Nasser. Numerical modeling of re- 
sponse of monolithic and bilayer plates to impulsive loads. International Journal of Impact Engineering, 37(1):90-102, 2010.

[17] M.R. Amini, J. Isaacs, and S. Nemat-Nasser. Investigation of effect of polyurea on response of steel plates to impulsive loads in direct pressure-pulse experiments. Mechanics of Materials, 42(6):628-639, 2010.

[18] M.R. Amini, J. Isaacs, and S. Nemat-Nasser. Experimental investigation of response of monolithic and bilayer plates to impulsive loads. International Journal of Impact Engineering, 37(1):82-89, 2010.

[19] V.S. Deshpande, A. Heaver, and N.A. Fleck. An underwater shock simulator. Proceedings of the Royal Society A, 462(2067):1021-1041, 2006.

[20] N.A. Fleck and V.S. Deshpande. The resistance of clamped sandwich beams to shock loading. Journal of Applied Mechanics, 71(3):1-16, 2004.

[21] E. Ferri, E. Antinucci, M.Y. He, J.W. Hutchinson, F.W. Zok, and A.G. Evans. Dynamic buckling of impulsively loaded prismatic cores. Journal of Mechanics of Materials and Structures, 1(8):1345-1365, 2006.

[22] S. Lee, F. Barthelat, J.W. Hutchinson, and H.D. Espinosa. Dynamic failure of metallic pyramidal truss core materials: Experiments and modeling. International Journal of Plasticity, 22(11):2118-2145, 2006.

[23] D.D. Radford, G.J. McShane, V.S. Deshpande, and N.A. Fleck. Dynamic compressive response of stainless-steel square honeycombs. Journal of Applied Mechanics, 74(4):658-667, 2007.

[24] Z. Xue and J.W. Hutchinson. Crush dynamics of square honeycomb sandwich 
cores. International Journal for Numerical Methods in Engineering, 65(13):2221$2245,2006$.

[25] M.T. Tilbrook, D.D. Radford, V.S. Deshpande, and N.A. Fleck. Dynamic crushing of sandwich panels with prismatic lattice cores. International Journal of Solids and Structures, 44(18-19):6101-6123, 2007.

[26] G.J. McShane, S.M. Pingle, V.S. Deshpande, and N.A. Fleck. Dynamic buckling of an inclined structure. International Journal of Solids and Structures, 49(1920):2830-2838, 2012.

[27] V.S. Deshpande and N.A. Fleck. High strain rate compressive behaviour of aluminium alloy foams. International Journal of Impact Engineering, 24(3):277$298,2000$.

[28] P.J. Tan, S.R. Reid, J.J. Harrigan, Z. Zou, and S. Li. Dynamic compressive strength properties of aluminium foams. Part I: Experimental data and observations. Journal of the Mechanics and Physics of Solids, 53(10):2174-2205, 2005.

[29] A. Vaziri and J.W. Hutchinson. Metal sandwich plates subject to intense air shocks. International Journal of Solids and Structures, 44(6):2021-2035, 2007.

[30] F. Zhu, Z. Wang, G. Lu, and G. Nurick. Some theoretical considerations on the dynamic response of sandwich structures under impulsive loading. International Journal of Impact Engineering, 37(6):625-637, 2010.

[31] G.J. Dvorak and A.P. Suvorov. Protection of sandwich plates from low-velocity impact. Journal of Composite Materials, 40(15):1317-1331, 2005.

[32] Y.A. Bahei-El-Din, G.J. Dvorak, and O.J. Fredricksen. A blast-tolerant sandwich 
plate design with a polyurea interlayer. International Journal of Solids and Structures, 43(25-26):7644-7658, 2006.

[33] Y.A. Bahei-El-Din and G.J. Dvorak. Wave propagation and dispersion in sandwich plates subjected to blast loads. Mechanics of Advanced Materials and Structures, 14(6):465-475, 2007.

[34] A. Yehia, Y.A. Bahei-El-Din, and G.J. Dvorak. Enhancement of blast resistance of sandwich plates. Composites B, 39(1):120-127, 2008.

[35] S. A. Tekalur, A. Shukla, and K. Shivakumar. Blast resistance of polyurea based layered composite materials. Composite Structures, 84(3):271-281, 2008.

[36] N. Gardner, E. Wang, P. Kumar, and A. Shukla. Blast mitigation in a sandwich composite using graded core and polyurea interlayer. Experimental Mechanics, 52(2):119-133, 2011.

[37] M.Z. Hassan, Z.W. Guan, W.J. Cantwell, G.S. Langdon, and G.N. Nurick. The influence of core density on the blast resistance of foam-based sandwich structures. International Journal of Impact Engineering, 50:9-16, 2012.

[38] G.E. Morgan. US Patent 3609764 - Energy absoring and sizing means for helmets, 1971.

[39] V. Ponomarev and I. Ponomaryova. US Patent 7017705 - Blast compression wave absorbing device, 2006.

[40] S. Zhuang, G. Ravichandran, and D.E. Grady. An experimental investigation of shock wave propagation in periodically layered composites. Journal of the Mechanics and Physics of Solids, 51(2):245-265, 2003. 
[41] V.F. Nesterenko. Shock (blast) mitigation by "soft" condensed matter. In Materials Research Society Symposium Proceedings, volume PROC-759-MM4.3, 2002.

[42] W.F. Hartman, B.A. Boughton, and M.E. Larsen. Blast mitigation capabilities of aqueous foam. Technical Report SAND2006-0533, Sandia National Laboratories, Albuquerque, NM 87185, USA, 2006.

[43] R.M. Allen, D.J. Kirkpatrick, A.W. Longbottom, A.M. Milne, and N.K. Bourne. Experimental and numerical study of free-field blast mitigation. AIP Conference Proceedings, 706(1):823-826, 2004.

[44] B.E. Gelfand, M.V. Silnikov, A.I. Mikhailin, and A.V. Orlov. Attenuation of blast overpressures from liquid in an elastic shell. Combustion, Explosion and Shock Waves, 37(5):607-612, 2001.

[45] A. Leonard and C. Daraio. Stress wave anisotropy in centered square highly nonlinear granular systems. Physical Review Letters, 108(21):1-4, 2012.

[46] V.F. Nesterenko. Dynamics of Heterogeneous Materials. Springer-Verlag, New York, NY, USA, 2001.

[47] C. Daraio, V.F. Nesterenko, E. Herbold, and S. Jin. Energy trapping and shock disintegration in a composite granular medium. Physical Review Letters, 96(5):14, 2006.

[48] B.R. Schimizze, S.F. Son, R. Goel, A.P. Vechart, and L.R. Young. An experimental and numerical study of blast induced shock wave mitigation in sandwich structures. Applied Acoustics, 74(1):1-9, 2013.

[49] H.N.G. Wadley, K.P. Dharmasena, M.Y. He, R.M. McMeeking, A.G. Evans, 
T. Bui-Thanh, and R. Radovitzky. An active concept for limiting injuries caused by air blasts. International Journal of Impact Engineering, 37(3):317-323, 2010.

[50] M. Grujicic, G. Arakere, and T. He. Material-modeling and structural-mechanics aspects of the traumatic brain injury problem. Multidiscipline Modelling in Materials and structures, 6:335-363, 2010.

[51] M. Grujicic, W.C. Bell, B. Pandurangan, and T. He. Blast-wave impactmitigation capability of polyurea when used as helmet suspension-pad material. Materials and Design, 31(9):4050-4065, 2010.

[52] L. Zhang, R. Makwana, and S. Sharma. Brain response to primary blast wave using validated finite element models of human head and advanced combat helmet. Frontiers in neurology, 4(88):1-12, 2013.

[53] S.G. Ganpule, L. Gu, A.L. Alai, and N. Chandra. Role of helmet in the mechanics of shock wave propagation under blast loading conditions. Computer Methods in Biomechanics and Biomedical Engineering, 15(11):1233-1244, 2012.

[54] D. Stewart, L.R. Young, R. Goel, G. Christou, and M.D. Gilchrist. Evaluating the performance of helmet linings incorporating fluid channels. Journal of ASME International, 7(10):1-7, 2010.

[55] J. Kimberley, J. Lambros, I. Chasiotis, J. Pulskamp, R. Polcawich, and M. Dubey. Mechanics of energy transfer and failure of ductile microscale beams subjected to dynamic loading. Journal of the Mechanics and Physics of Solids, 58(8):1125-1138, 2010.

[56] S.J. Stritch. Shearing of nerve fibres as a cause of brain damage due to head injury: A pathological study of twenty cases. Lancet, 278:443-448, 1961. 
[57] N.C. Nevin. Neuropathological changes in the white matter following head injury. Journal of Neuropathology and Experimental Neurology, 26:77-84, 1967.

[58] V.E. Johnson, W Stewart, and Smith D.H. Axonal pathology in traumatic brain injury. Experimental Neurology, 246:35-43, 2013.

[59] A.H.S. Holbourn. Mechanics of head injuries. Lancet, 242:438-441, 1943.

[60] E.S. Gurdjian, H.R. Lissner, F.R. Latimerr, B.F. Haddad, and J.E. Webster. Quantitative determination of acceleration and intercranial pressure in experimental head injury. Neurology, 3:417-423, 1953.

[61] W.N. Hardy, T.B. Khalil, and A.I King. Literature review of head injury biomechanics. International Journal of Impact Engineering, 15(4):561-586, 1994.

[62] J. S. Ruan and P. Prasad. Coupling of a finite element human head model with a lumped parameter hybrid III dummy model: Preliminary results. Journal of Neurotrauma, 12(4):725-734, 2007.

[63] L.E. Goldstein, A.M. Fisher, C.A. Tagge, X.L. Zhang, L. Velisek, J.A. Sullivan, C. Upreti, J.M. Kracht, M. Ericsson, M.W. Wojnarowicz, C.J. Goletiani, G.M. Maglakelidze, N. Casey, J.A. Moncaster, O. Minaeva, R.D. Moir, C.J. Nowinski, R.A. Stern, R.C. Cantu, J. Geiling, J.K. Blusztajn, B.L. Wolozin, T. Ikezu, T.D. Stein, A.E. Budson, N.W. Kowall, D. Chargin, A. Sharon, S. Sudad, G.F. Hall, W.C. Moss, R.O. Cleveland, R.E. Tanzi, P.K. Stanton, and A.C. McKee. Chronic traumatic encephalopathy in blast-exposed military veterans and a blast neurotrauma mouse model. Science Translational Medicine, 4(134):1-14, 2012.

[64] J.M. Meythaler, J.D. Peduzzi, E. Eleftheriou, and T.A. Novack. Current con- 
cepts: Diffuse axonal injuryassociated traumatic brain injury. Archives of Physical Medicine and Rehabilitation, 82(10):1461-1471, 2001.

[65] H.W. Henn. Crash tests and head injury criteria. Teaching Mathematics and its Applications, 17(4):162-170, 1998.

[66] S.P. Broglio, J.T. Eckner, T. Surma, and J.S. Kutcher. Post-concussion cognitive declines and symptomatology are not related to concussion biomechanics in high school football players. Journal of Neurotrauma, 28(10):1-8, 2011.

[67] S.P. Broglio, B. Schnebel, J.J. Sosnoff, S. Shin, X. Fend, X. He, and J. Zimmerman. Biomechanical properties of concussions in high school football. Medicine and Science in Sports and Exercise, 42(11):2064-2071, 2010.

[68] R.M. Greenwald, J.T. Gwin, J.J. Chu, and J.J. Crisco. Head impact severity measures for evaluating mild Traumatic Brain Injury risk explosure. Neurosurgery, 62(4):789-798, 2008.

[69] H. Kolsky. Stress Waves in Solids. Dover Publications, New York, NY, USA, 2nd edition, 2012.

[70] R.C. Hibbeler. Engineering Mechanics: Statics and Dynamics. Macmillan Publishing Company, New York, NY, USA, 5th edition, 1989.

[71] A.S. Wineman and K.R. Rajagopal. Mechanical Response of Polymers: An Introduction. Cambridge University Press, Cambridge, UK, 2000.

[72] I. Alig, F. Stieber, and S. Wartewig. Ultrasonic examination of the dynamic glass transition in amorphous polymers. Journal of Non-Crystalline Solids, 131133:808-811, 1991. 
[73] C.M. Rowland and R. Casalini. Effect of hydrostatic pressure on the viscoelastic response of polyurea. Polymer, 48:5747-5752, 2007.

[74] R.B. Bogoslovov, C.M. Rowland, and R.M Gamache. Impact-induced glass transition in elastomeric coatings. Applied Physics Letters, 90(221910):1-3, 2007.

[75] A.J. Hsieh, T.L. Chantawansri, W. Hu, K.E. Strawhecker, D.T. Casem, J.K. Eliason, K.A. Nelson, and E.M. Parsons. New insight into microstrcuture-mediated segmental dynamics in select model poly(urethane urea) elastomers. Polymer, 55:1883-1892, 2014.

[76] ABAQUS version 6.8-1, User Documentation, Dassault Systems, 2008.

[77] J.W. Srutt. On the equation of the stability of the flow of liquids. Philosophical Magazine, 34:59-70, 1892.

[78] A Vaschy. Sur les lois de similitude en physique. Annales Telegraphiques, 19:25$28,1892$.

[79] E. Buckingham. On physically similar systems: illustrations of the use of dimensional equations. Physical Reviews, 4(4):345-376, 1914.

[80] M.L. Williams, R.F. Landel, and L.D. Ferry. The temperature dependence of relaxation mechanisms in amorphous polymers and other glass-forming liquids. Journal of the American Chemical Society, 77(14):3701-3707, 1955.

[81] D. Denny-Brown and W.R. Russell. Experimental cerebral concussion. Brain, 64(2-3):93-164, 1941.

[82] MATLAB SIMULINK R2011a, MathWorks, 2011. 


\section{Appendix A.}

There is a general tendency in the brain injury literature to measure the acceleration of the head as a proxy for the stresses acting on the brain $[63,64,65,66,67,68]$. However, it was recognized more than 70 years ago [59] that this is valid only for impacts of long duration. For short impacts and blasts, it is the change in velocity of the head that indicates the level of the stresses in the brain. The head is a complex dynamical system, so the force transmitted to the brain cannot simply be taken to be the force applied to the skull. The purpose of this Appendix is to use a very simple dynamical system to illustrate this point, since identifying the correct mechanics is crucial for evaluating design concepts for armor.

As shown in Fig. A.1, a delicate target supported by a structural support is modeled as two point masses coupled by two springs. The structural support is represented by a mass $m_{1}$ that is attached to a rigid foundation by a spring of stiffness $k_{1}$. The force pulse transmitted from the blast, either directly or as modified by passage through the armor, $F(t)$, is applied to this structural support. In a simple model of the head, this structural support is the skull, and the spring represents the stiffness of the neck. The importance of this spring has been demonstrated experimentally by showing that bracing the neck decreases the severity of brain damage $[63,81]$. The delicate target is represented by a mass $m_{2}$ that is attached to the structural support by a spring of stiffness $k_{2}$. For example, in a simple model of the head, the target is the brain, and the spring represents the cerebrospinal fluid.

The load that can cause damage to the target is given by

$$
F_{2}(t)=k_{2}\left[x_{1}-x_{2}\right]=m_{2} \ddot{x}_{2}
$$




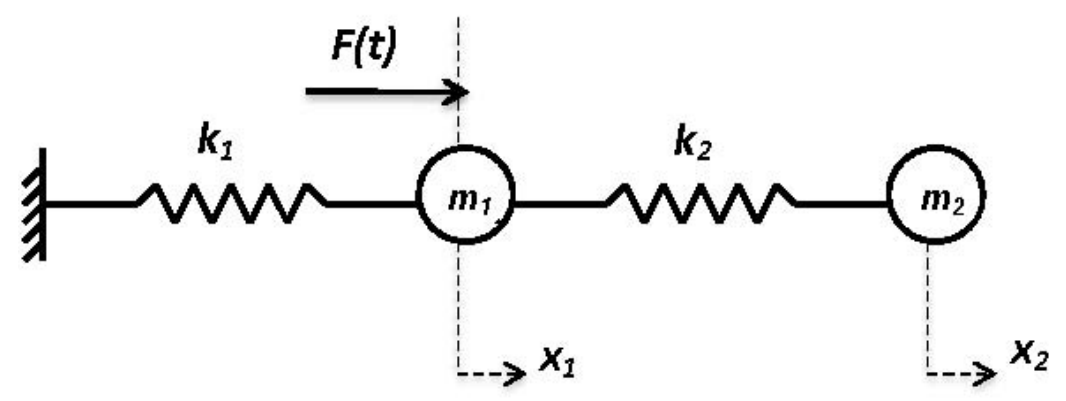

Figure A.1: Dynamic model of a structural support of mass $m_{1}$ attached to a rigid foundation by a spring of stiffness $k_{1}$, and coupled to a delicate target of mass $m_{2}$ through a spring of stiffness $k_{2}$. A pressure force $F(t)$ is transmitted to the structure either directly from the blast, or through the armor.

where $x_{2}$ is the displacement of the target, and $x_{1}$ is the displacement of the structure (Fig. A.1). The relationship between the force imposed on the structure, $F(t)$, and the force acting on the target, $F_{2}(t)$, is given by

$$
F_{2}(t)=F(t)-\left[m_{1} \ddot{x}_{1}+k_{1} x_{1}\right]
$$

A calculation of the pressure that acts on the target is obtained by solving these two equations, subject to an assumption that the structure and target are assumed to be initially at rest $\left(x_{1}=x_{2}=\dot{x}_{1}=\dot{x}_{2}=0\right)$, and the transmitted force is a decaying triangular pulse with a peak force $F_{t}$ and a duration of $t_{t}$.

A non-dimensional analysis of this problem indicates that the maximum force acting on the target and on the supporting structure are both of the form

$$
\frac{P_{i_{\max }}}{P_{t}}=f\left(\frac{m_{1}}{m_{2}}, \frac{k_{1}}{k_{2}}, \sqrt{\frac{k_{2}}{m_{2}}} t_{t}\right),
$$

where $i=1$ indicates the supporting structure and $i=2$ indicates the delicate target. A MATLAB SIMULINK [82] calculation was performed to show how $F_{1_{\max }}$ and $F_{2_{\max }}$ vary with $\sqrt{k_{2} / m_{2}} t_{t}$ for a range of masses and spring constants. 


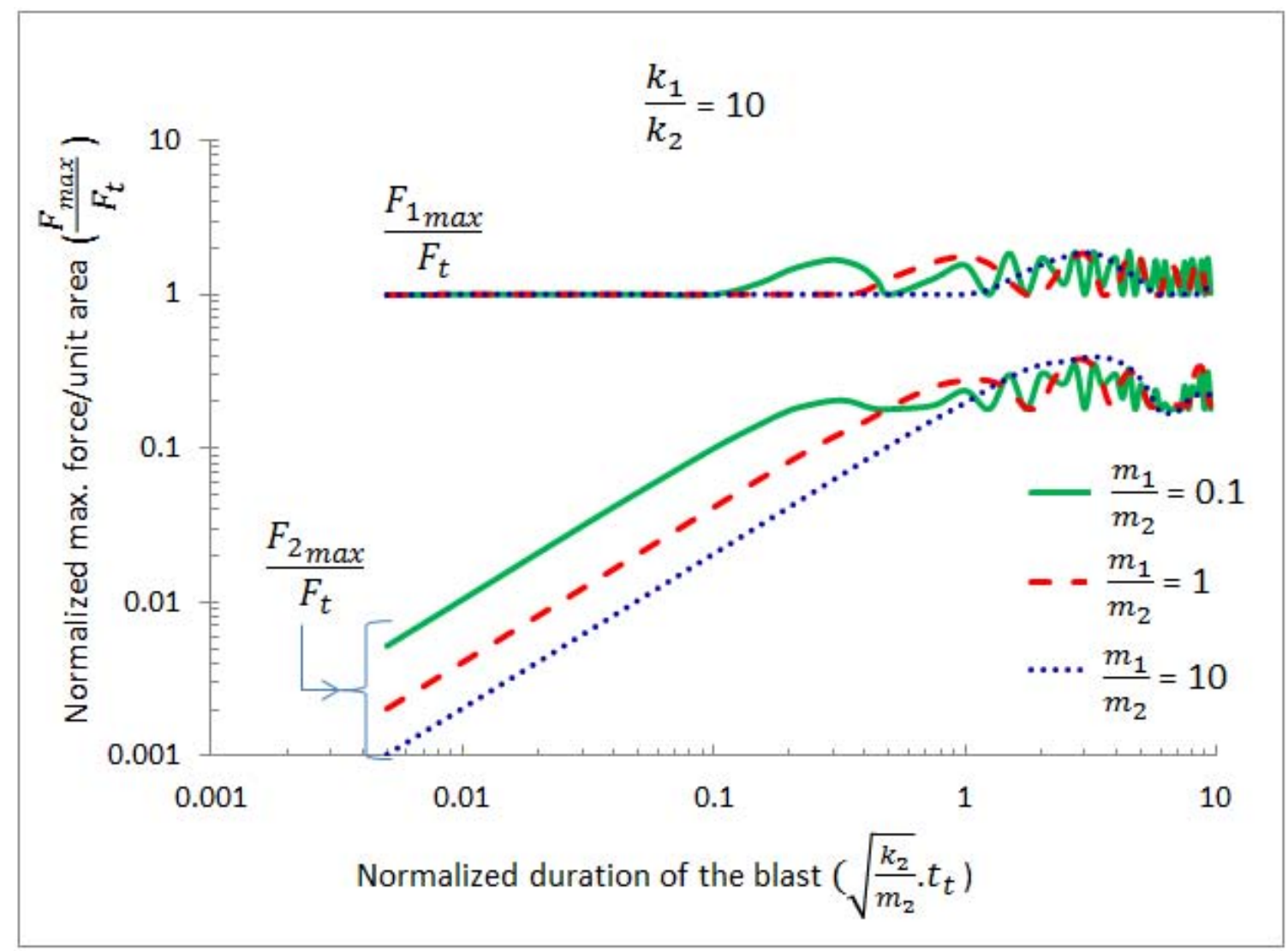

Figure A.2: Maximum pressure exerted on the structure, $F_{1_{\max }} / F_{t}$, and on the delicate target, $F_{2_{\max }} / F_{t}$, as a function of normalized time, $\sqrt{k_{2} / m_{2}} t_{t}$. The maximum pressure on the structure is relatively constant and approximately equal to the amplitude of the transmitted pressure. The maximum pressure on the target depends on the transmitted impulse when the transmitted pulse is shorter than the natural period of the target. It depends on the amplitude of the transmitted pressure only when the pulse is longer than the natural period of the target.

Figure A.2 shows how the maximum force acting on the supporting structure is relatively constant and approximately equal to $P_{t}$. This is what would be measured by placing an accelerometer on the supporting structure. However, it can also be seen from this figure that the maximum force on the target depends on the transmitted impulse when the duration of the pulse is shorter than the natural period of the target. The maximum force on the target only depends on the amplitude of the transmitted force when the duration of the pulse is longer than the natural period of the target. 
Although this is a relatively simple dynamical model, there are several important conclusions about protecting targets from damage arising from blast or impact. The first is that the maximum force on the target does not necessarily correlate with the maximum force applied to the structure. A practical implication of this is that a simple measurement of maximum acceleration of a skull may give no indication of the force that a brain experiences. Acceleration measurements are useful as part of a complete time-history record. The second is that the correct protective strategy depends on the duration of the blast or impact compared to the natural frequency of the target one is trying to protect. If the duration of the impact is long, then it is the amplitude of the transmitted pressure that has to be reduced. This can be done by mismatch of impedance. If the duration of the impact is short, then it is the transmitted impulse that has to be minimized. 